\title{
ON THE PERFORMANCE OF RANDOM-PROJECTION-BASED DIMENSIONALITY REDUCTION FOR ENDMEMBER EXTRACTION
}

\author{
Qian Du, James E. Fowler \\ Department of Electrical and Computer Engineering \\ Mississippi State University, Mississippi State, MS 39762, USA \\ $\{\mathrm{du}$, fowler\}@ece.msstate.edu
}

\begin{abstract}
In this paper, we investigate the use of randomprojection-based dimensionality reduction for hyperspectral endmember extraction. It is data-independent and computationally more efficient than other widely used dimensionality reduction methods, such as principal component analysis and maximum noise fraction transform. Based on the preliminary result, random-projection-based dimensionality reduction is capable of providing better endmembers after effective decision fusion.
\end{abstract}

Index Terms - dimensionality reduction, random projection, endmember extraction, hyperspectral imagery

\section{INTRODUCTION}

Random projection (RP) is a computationally efficient and data-independent method for dimensionality reduction (DR). The transformation matrix of RP generally includes Gaussian distributed random vectors, which are mutually orthogonal unit vectors. Theoretical results indicate that it can well preserve distances among data points as well as the structure of data cloud [1-3]. Recently, RP is of great interest because the mathematical theory of popular compressed sensing shows that sparsely representable signals can be recovered exactly from RP [4].

The traditional DR methods, such as principal component analysis (PCA) and maximum noise fraction (MNF) transform, are data-dependent and involve burdensome computations. For a hyperspectral image with $N$ pixels and $L$ bands, PCA needs $L^{2} N$ multiplications for covariance matrix calculation, $O\left(L^{3}\right)$ for eigen-decomposition, and $K L N$ multiplications for the transform (with $K$ principal components (PCs)); MNF needs the computations for noise covariance matrix estimation and noise whitening, in addition to those in the original PCA. If RP is adopted, tremendous savings can be achieved. If the original data is reduced to $K$-dimensional, the computation time involved in the random matrix generation is only $O\left(K^{2} L\right)$ including the expensive Gram-Schmidt orthogonalization process; it can be further reduced to $O(K L)$ if using uniformly distributed random variables without orthogonalization. Note that, for a hyperspectral remote sensing image, $K<L<<N$.

Endmember extraction is an important step in spectral mixture analysis for hyperspectral imagery. Usually, DR is a preprocessing step for endmember extraction, which can not only save computational time but also improve the performance. The performance improvement comes from the automated removal of spectrally trivial variation in pixel signatures [5]. In general, MNF is preferred since it can compact the major data information in terms of signal-tonoise ratio, a better criterion than variance used in PCA.

In this paper, we evaluate the performance of RP-based DR for endmember extraction and compare it with the PCAor MNF-based DR. Widely used endmember extraction algorithms, such as N-FINDR [6] and VCA [7], are examined. N-FINDR is a parallel algorithm, finding all the endmembers simultaneously; VCA is a sequential algorithm, extracting endmembers one after another. Many endmember extraction algorithms assume that pure pixels exist in an image scene; thus, they actually find distinctive pixels as endmembers. Here, we limit our discussion on this type of algorithms, although there are algorithms in the literature without pure pixel assumption.

\section{METHODOLOGY}

\subsection{Proposed Algorithm}

To reduce the data dimensionality from $L$ to $K$ using RP, a random matrix of size $K \times L$ needs to be generated. Here, we use Gaussian random variables and column vectors are mutually orthogonal unit vectors, although these may not be necessary. Then an original endmember extraction algorithm is applied to randomly projected data. Since RP is data-independent and does not optimize any criterion, the extracted endmembers may not be the best set for a specific run. However, from multiple runs with different random matrices, a similar or even better result can be generated, compared to the one from PCA- or MNF-based DR (which has a single run only). This is due to the phenomenon that a 
distinctive pixel generally remains distinctive [8]. However, for a specific RP, a distinctive pixel may become less distinctive; thus, multiple runs are needed to find all the distinctive pixels, in particular, those are not significantly distinctive in the original data.

The entire algorithm can be illustrated with the diagram in Fig. 1. Different fusion methods can be used to finalize endmembers. The simplest one is to find those endmembers that are extracted by $m$ times out of $n$ runs. The ratio of $m / n$ to be chosen is related to $K / L$. For very distinctive endmembers, both $m / n$ and $K / L$ can be very small. To same computational time, we may find those very distinctive endmembers first using a small $K / L$, then project the data onto the orthogonal subspace of these endmembers to remove them. Less distinctive endmembers can be extracted with a larger $K / L$ using the orthogonal-projected data.

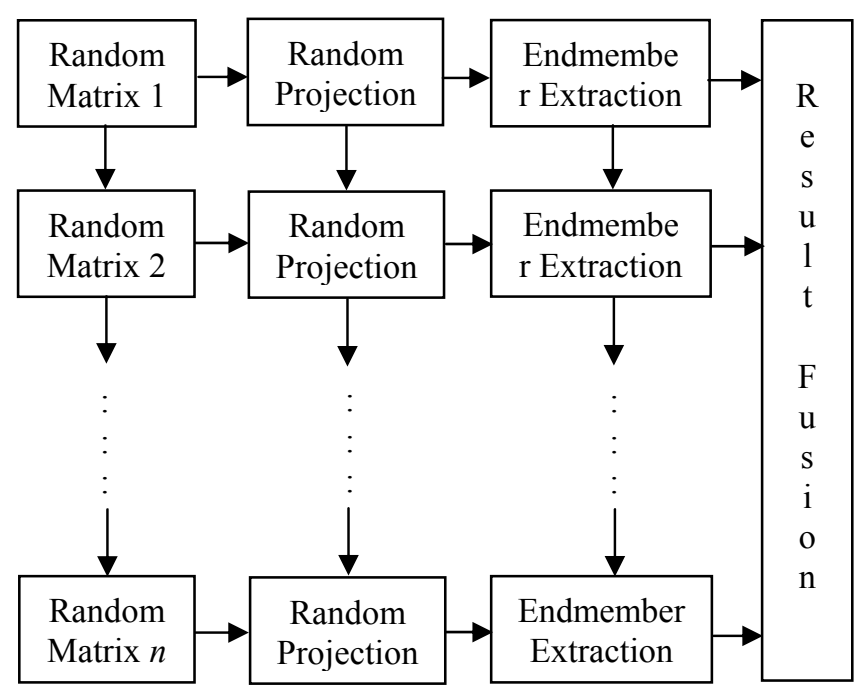

Figure 1. The proposed approach for endmember extraction.

\subsection{Performance Evaluation}

When a spectral library is known, extracted endmembers can be simply compared with true endmember signatures using spectral angle. If no ground truth about endmember signatures is available, the volume of the simplex formed by extracted endmembers is calculated, and the set resulting in a larger volume is considered as a better one since it includes more distinctive endmembers; or, the pairwise spectral angles are computed, and the set providing the larger average spectral angle is claimed to be the better one.

\section{EXPERIMENTS}

AVIRIS Lunar Lake image data of size $200 \times 200$ with 158 bands (after removing bad bands) was used in the experiments. There are six endmembers: Cinder (C), Playa

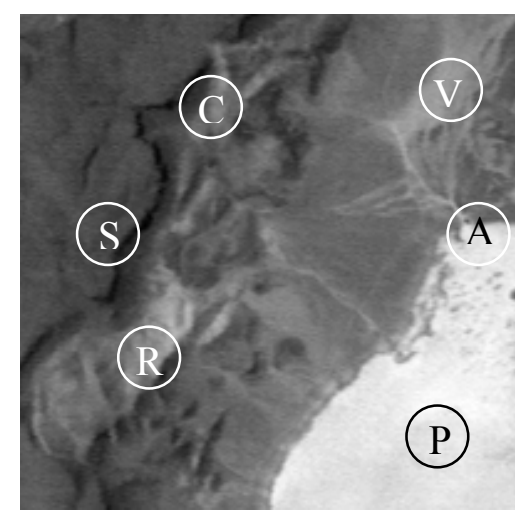

Figure 2. An AVIRIS lunar lake subscene including six endmemebrs named as Cinder (C), Playa Lake (P), Rhyolite (R), Vegetation (V), Shade (S), and an Anomaly (A).

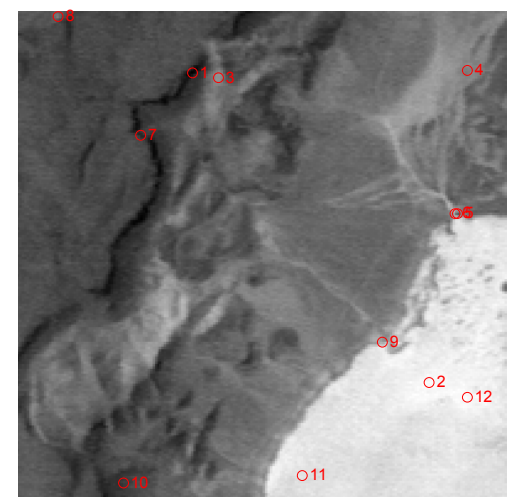

Figure 3. The endmembers extracted by the VCA algorithm from the original data.

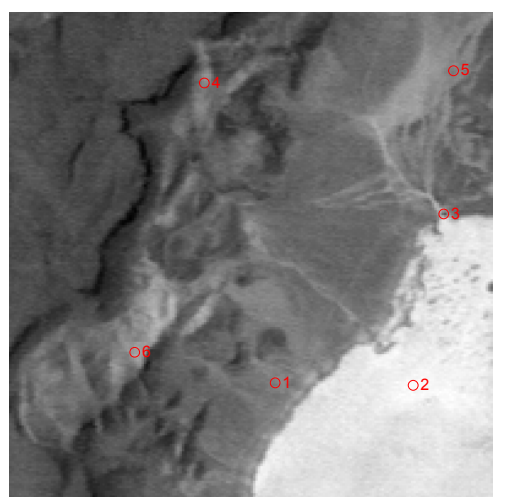

Figure 4. The endmembers extracted by the VCA algorithm from the PCA-transformed data (with 6 PCs).

Lake (P), Rhyolite (R), Shade (S), Vegetation (V), and an Anomaly (A). Their approximate spatial locations are marked in Fig. 2.

For this image data, PCA results were better than MNF results. So RP was compared with PCA only. Fig. 3 shows the VCA-extracted endmembers using the original data. We can see that the Rhyolite endmember could not be extracted even when the number of endmembers was increased to 12 . 


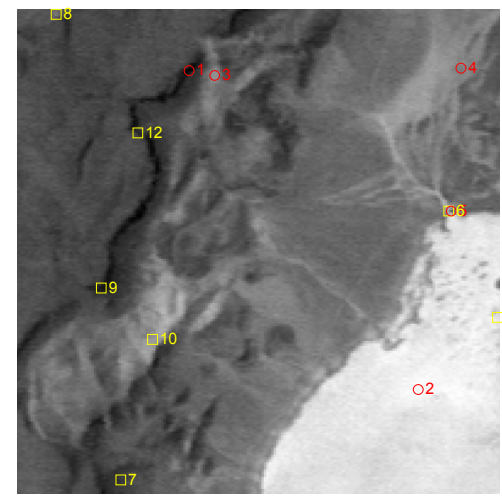

Figure 5. The endmembers extracted by the VCA algorithm from the RP-transformed data (red circle: $K / L=$ 0.1 ; yellow square: $K / L=0.6$ ).

TABLE I

COMPARISON OF VCA RESULTS USING PCA AND RP FOR DIMENSIONALITY REDUCTION

\begin{tabular}{|c|c|c|}
\hline $\begin{array}{c}\text { Volume } \\
\left(\times 10^{14}\right)\end{array}$ & 3.02 & 7.53 \\
\hline Mean & $10.4^{\circ}$ & $13.9^{\circ}$ \\
\hline Std. & $5.6^{\circ}$ & $6.0^{\circ}$ \\
\hline
\end{tabular}

Fig. 4 shows the result using the PCA-transformed data and all the six types of endmembers were extracted when the number of endmembers was 6 . This also demonstrated the advantage of using DR as a preprocessing step.

Fig. 5 is the result from RP. The endmembers for Shade, Playa Lake, Cinder, Vegetation, and Anomaly could be easily found with $K / L=0.1$. After the data was projected onto their orthogonal subspace, the Rhyolite endmember could be extracted with $K / L=0.6$. In the experiments, the number of runs $n=20$, and endmembers were finalized if they were extracted more than $m=5$ times.

Table I lists the simplex volumes constructed by endmembers extracted when PCA and RP were employed for DR. The endmember set from RP had a larger volume. The pairwise spectral angles were also calculated, and the set from RP had greater mean and standard deviation.

N-FINDR was also used for endmember extraction. To avoid the result change from random initials, N-FINDR was initialized using the VCA result [9]. Fig. 6 marked the endmembers from using 5 PCs. Table II lists the simplex volume and spectral angle before and after N-FINDR. After applying N-FINDR, the simplex volume was significantly increased. Compared Fig. 6 with Fig. 4, we can see that endmembers for Cinder, Rhyolite, and Shade were changed.

For RP-based N-FINDR, $K / L$ was varied from 0.1 to 0.6. The fused result from 20 runs was illustrated in Fig. 7, where endmembers for Cinder, Rhyolite, and Shade were further changed. Quantitative assessment was listed in Table III. Simplex volumes were larger than that from PCA and

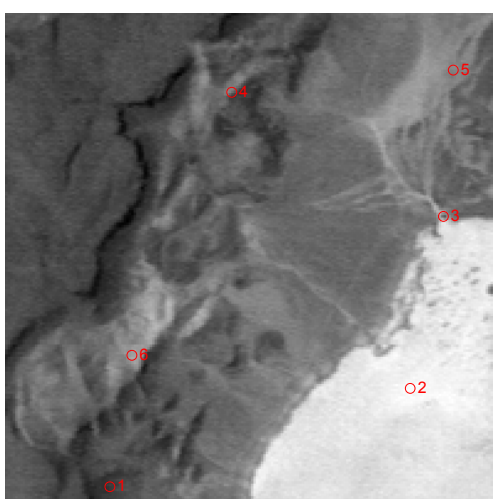

Figure 6. The endmembers extracted by the N-FINDR algorithm from the PCA-transformed data (with 5 PCs).

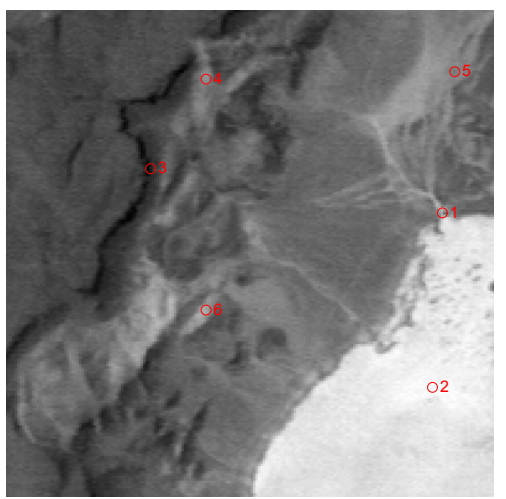

Figure 7. The endmembers extracted by the N-FINDR algorithm from the RP-transformed data $(K / L=0.1)$.

TABLE II

QUALITY ASSESSMENT FOR ENDMEMBERS EXTRACTED FROM NFINDR AFTER PCA-BASED DIMENSIONALITY REDUCTION

\begin{tabular}{|c|c|c|}
\hline & $\begin{array}{c}\text { Before } \\
\text { N-FINDR }\end{array}$ & $\begin{array}{c}\text { After } \\
\text { N-FINDR }\end{array}$ \\
\hline $\begin{array}{c}\text { Volume } \\
\left(\times 10^{14}\right)\end{array}$ & 3.02 & 5.24 \\
\hline Mean & $10.4^{\circ}$ & $10.1^{\circ}$ \\
\hline Std. & $5.6^{\circ}$ & $4.1^{\circ}$ \\
\hline
\end{tabular}

TABLE III

QUALITY ASSESSMENT FOR ENDMEMBERS EXTRACTED FROM NFINDR AFTER RP-BASED DIMENSIONALITY REDUCTION

\begin{tabular}{|c|c|c|c|c|c|c|}
\hline$K / L$ & 0.1 & 0.2 & 0.3 & 0.4 & 0.5 & 0.6 \\
\hline $\begin{array}{c}\text { Volume } \\
\left(\times 10^{14}\right)\end{array}$ & 6.31 & 5.79 & 6.13 & 5.82 & 6.10 & 6.18 \\
\hline Mean & $12.2^{\circ}$ & $11.3^{\circ}$ & $12.9^{\circ}$ & $13.2^{\circ}$ & $12.6^{\circ}$ & $12.7^{\circ}$ \\
\hline Std. & $5.3^{\circ}$ & $5.2^{\circ}$ & $5.6^{\circ}$ & $5.7^{\circ}$ & $5.1^{\circ}$ & $5.5^{\circ}$ \\
\hline
\end{tabular}

endmembers became more different. Interestingly, using a small $K / L$ did not necessarily provide a worse result. However, more runs may be needed for a small $K / L$ in order to extract the endmembers of interest. 


\section{CONCLUSION}

In this paper, we propose RP-based dimensionality reduction for endmember extraction. It is data-independent and computationally more efficient than other widely used dimensionality result methods, such as PCA and MNF. Based on preliminary result, RP-based dimensionality reduction is capable of providing better endmembers after multiple runs and effective decision fusion. As the future work, the best fully automated fusion strategy is to be investigated.

\section{ACKNOWLEDGMENT}

This research is supported in part by National Geospatial-Intelligence Agency.

\section{REFERENCES}

[1] S. Dasgupta, "Experiments with random projections," In Proceedings of the Sixteen Conference on Uncertainty in Artificial Intelligence, pp. 143-151, 2000.

[2] E. Bingham and H. Mannila, "Random projection in dimensionality reduction: Applications to image and text data," in Proceedings of ACM Conference on Knowledge Discovery and Data Mining, pp. 245-250, 2001.
[3] D. Achlioptas, "Database-friendly random projections," In Proceedings of ACM Symposium on the Principles of Database Systems, pp. 271-281, 2001.

[4] E. J. Candes and M. B. Wakin, "An introduction to compressive sampling," IEEE Signal Processing Magazine, vol. 25, no. 2, pp. 21-30, Mar. 2008.

[5] Q. Du, N. Raksuntorn, N. H. Younan, and R. L. King, "Endmember extraction for hyperspectral imagery," Applied Optics, vol. 47, no. 28, pp. F77-F84, Oct. 2008.

[6] M. E. Winter, "N-FINDR: an algorithm for fast autonomous spectral end-member determination in hyperspectral data," Proc. SPIE 3753, pp. 266-275, 1999.

[7] J. M. P. Nascimento and J. M. Bioucas Dias, "Vertex component analysis: a fast algorithm to unmix hyperspectral data," IEEE Transactions on Geoscience and Remote Sensing, vol. 43, no. 4, pp. 898-910, Apr. 2005.

[8] J. E. Fowler, Q. Du, W. Zhu, and N. H. Younan, "Classification performance of random-projectionbased dimensionality reduction of hyperspectral imagery," Proceedings of IEEE Geoscience and Remote Sensing Symposium, Cape Town, South Africa, Jul. 2009.

[9] A. Plaza and C.-I Chang, "Impact of initialization on design of endmember extraction algorithms," IEEE Trans. Geosci Remote Sensing, vol. 44, no. 11, pp. 3397-3407, Nov. 2006. 